\title{
Prophylaxe der Rückenmarkischämie bei Therapie von Aortenaneurysmen
}

Konstantin von Aspern, Christian D. Etz

\begin{abstract}
Die moderne Therapie des thorakoabdominellen Aortenaneurysmas birgt ein immer noch erhebliches Risiko: die iatrogene ischämische Schädigung des Rückenmarks mit der Konsequenz der permanenten Paraplegie. Dieser Beitrag schildert die aktuell verfügbaren unterstützenden Therapiemaßnahmen zur Rückenmarkprotektion und stellt ein neues klinisches Präventionsverfahren vor, das experimentell nahezu $100 \%$-igen Schutz erzielt hat.
\end{abstract}

\section{Thorakoabdominelles}

\section{Aortenaneurysma}

Thorakoabdominelle Aortenaneurysmen sind selten die geschätzte Inzidenz liegt bei weniger als 6 Fällen auf 100000 Einwohner pro Jahr [1]. Ihre sozioökonomischen und individuellen Folgen sind jedoch gravierend. Sie entstehen sowohl auf dem Boden von degenerativen und atherosklerotischen Gefäßwandveränderungen als auch aufgrund von Bindegewebserkrankungen und chronischen Aortendissektionen [1]. Diese unterliegen einer steigenden Tendenz in industrialisierten Ländern.

Das Risiko einer spontanen Komplikation (Einriss oder Ruptur) eines Aneurysmas steigt ab einem kritischen Aortendurchmesser exponentiell auch unter medikamentöser Blutdruckregulation. Deshalb ist eine Operation ab einem Durchmesser von $6 \mathrm{~cm}$ indiziert und bereits ab $5 \mathrm{~cm}$ im Falle von Bindegewebserkrankungen wie z.B. dem Marfan-Syndrom [2,3].

Aktuell konkurriert in der Therapie des thorakoabdominellen Aortenaneurysmas der offen-chirurgische Ersatz zunehmend mit der endovaskulären Stentgraft-Implantation. Dabei gilt bei ausgedehnten thorakoabdominellen Aortenaneurysmen (Crawford-Typ II) der operative Ersatz nach wie vor als Goldstandard [2]. Hingegen werden isolierte Deszendenzaneurysmen meist standardmäßig endovaskulär behandelt, wenn keine Bindegewebserkrankung vorliegt.

$\begin{array}{ll}\text { ABKÜRZUNGEN } \\ \text { CnNIRS } & \text { Kollateralnetzwerk-Nahinfrarotspektroskopie } \\ \text { CT } & \text { Computertomografie } \\ \text { DFG } & \text { Deutsche Forschungsgemeinschaft } \\ \text { INR } & \text { International normalized Ratio } \\ \text { MEP } & \text { motorisch evozierte Potenziale } \\ \text { MISACE } & \text { Minimally invasive segmental Artery Coil and } \\ & \text { Plug Embolization } \\ \text { MRT } & \text { Magnetresonanztomografie } \\ \text { PAPA_Artis } & \text { Paraplegia Prevention in aortic Aneurysm Repair } \\ & \text { by thoracoabdominal Staging with minimally- } \\ & \text { invasive segmental Artery Coil-Embolization } \\ \text { SSEP } & \text { (MISACE; EU Horizon 2020 Studie) } \\ \text { TASP } & \text { Somatosensibel evozierte Potenziale } \\ \text { TEVAR } & \text { Temporay Aneurysm Sac Perfusion } \\ & \text { Thoracic Endovascular Aortic Repair }\end{array}$

\section{Risiko des therapieassoziierten ischämischen Rückenmarkschadens}

\footnotetext{
Merke

Sowohl beim offen-chirurgischen Aortenersatz als auch in der endovaskulären Stent-Therapie (TEVAR [Thoracic Endovascular Aortic Repair]) ist das Risiko einer ischämischen Schädigung des Rückenmarks hoch. Die Konsequenz ist eine permanente Paraplegie.
}

Aktuelle klinische Studien beziffern das Risiko unabhängig vom gewählten Verfahren beim Crawford-Typ II mit 6\% bis 25\%, im Durchschnitt $18 \%$ [3].

Eine Optimierung des intra- und postoperativen Managements konnte das Risiko auch in wenigen hochspezialisierten Zentren nicht unter $5 \%$ senken [4]. Fol- 
gende Maßnahmen werden zu diesem Zweck u.a. eingesetzt:

- distale Perfusion mit und ohne Oxygenator an der extrakorporalen Zirkulation (Herz-Lungen-Maschine oder Biocircuit ${ }^{\circledR}$ )

- Hypothermie (milde: $33-35^{\circ} \mathrm{C}$, moderate: $28-32^{\circ} \mathrm{C}$, tiefe: $21-27^{\circ} \mathrm{C}$ und profunde: $<20^{\circ} \mathrm{C}$ Hypothermie)

- konsequente Liquordrainage (manuelle oder automatisierte)

- hochnormales Blutdruckmanagement entsprechend dem individuellen Ausgangswert

- Versorgung durch erfahrene Aorten-Teams

Hinsichtlich der Kausalität der Paraplegie ist der unmittelbare, durch eine intraoperative Minderperfusion ausgelöste ischämische Schaden von der verzögerten spinalen Ischämie zu unterscheiden.

\section{Merke}

Die verzögerte spinale Ischämie tritt meist zwischen dem 2. und 5. postoperativen Tag auf, kann jedoch auch erst Wochen nach dem Eingriff eintreten. Sie stellt neben pulmonalen Komplikationen das zentrale Problem in der postoperativen Nachsorge dar.

\section{Therapiekonzepte der Rückenmarkperfusion}

\section{Anatomisches versus physiologisches Modell der Rückenmarkperfusion}

Hinsichtlich der Prophylaxe und Therapie des ischämischen Rückenmarkschadens herrscht weiterhin erheblicher Dissens unter Experten. Auf der einen Seite gibt es Protagonisten, die historisch bedingt eine primär anatomische Reimplantation von Segmentarterien bevorzugen. Ihnen stehen jene gegenüber, die den Fokus auf eine ideale Perfusionsaugmentation z. B. durch optimierte Hämodynamik legen.

Vertreter des primär anatomischen Paradigmas gehen von Beschreibungen aus historischen Untersuchungen zur Rückenmarkperfusion [5] aus. Diese legen nahe, dass die Wiederherstellung bzw. Reimplantation möglichst vieler arterieller Zuflüsse über aortale Segmentarterien (insbesondere der A. Adamkiewicz, siehe unten) eine zentrale Bedeutung für die Verhinderung des ischämischen Rückenmarkschadens hat.

Diesem entgegen stehen neuere Untersuchungen, die die Offenheitsrate reimplantierter Segmentarterien fundamental infrage stellen [6]. Entsprechend dem rein physiologischen Modell zur Rückenmarkprotektion leisten folgende Maßnahmen zur Steigerung der effektiven Rückenmarkperfusion den zentralen Beitrag zur Verhinderung des ischämischen Schadens:
- adäquates Temperaturmanagement

- hochnormales systemisches Blutdruckmanagement

- konsequente Senkung des intrathekalen Druckes (mittels Liquordrainage)

\section{Neues Konzept der spinalen Blutversorgung}

Das Konzept der spinalen Blutversorgung beruhte seit dem späten 19. Jahrhundert auf den anatomischen Beschreibungen von Albert W. Adamkiewicz. Die nach ihm benannte prominente $A$. radicularis magna als größte anteriore Segmentarterie galt bis vor einigen Jahren als der allein ausschlaggebende Versorgungsweg für das Rückenmark [5]. Detaillierte Studien von Lazorthes u. Mitarb. zeigten seit 1970 bereits, dass die spinale Blutversorgung weitaus komplexer und vielfältiger ist als ursprünglich angenommen [7]. Diese Erkenntnisse wurden jedoch weitestgehend ignoriert und lange nicht konsequent weiter untersucht. Erst Etz und Griepp konnten durch intensive, systematische Forschungsarbeiten einen ganzheitlichen, neuen Ansatz der Rückenmarkversorgung erarbeiten, die gegenwärtig einen Paradigmenwechsel einleiten $[8,9]$.

Merke

Das Konzept des paraspinalen Kollateralnetzwerks hat gegenwärtig einen Paradigmenwechsel im Verständnis und in der klinischen Versorgung von Patienten mit Erkrankungen an der thorakoabdominellen Aorta zur Folge.

DEFINITION/SYNONYM

Das Kollateralnetzwerk entspricht einem extensiven arteriellen Netzwerk, das weitestgehend in der paraspinalen Muskulatur lokalisiert ist. Es geht von Abgängen der Aa. subclaviae bzw. mammariae und der A. hypogastrica sowie direkt aus aortalen Segmentarterien aus. Über Kollateralen verteilt es sowohl longitudinal als auch horizontal das Blut für das Rückenmark segmentübergreifend auf das intraspinale Netzwerk. So stellt es die Rückenmarkversorgung, auch bei Verlust segmentaler Perfusion, sicher ( $\triangleright$ Abb. 1).

Intraspinale, unmittelbar verfügbare Umgehungskreisläufe, die sich aus repetitiven, Willis-ähnlichen Mikronetzwerken zusammensetzen, sichern die Rückenmarkversorgung [10]. Experimentelle Untersuchungen konnten zeigen, dass es nach Segmentarterienverschluss zu Änderungen des Zuflusses und der regionalen Blutdruckunterschiede kommt. Das versetzt das Kollateralnetzwerk in die Lage, über Arteriogenese neuer und durch Kaliberänderung präformierter Arterien und Arteriolen eine robuste alternative Blutversorgung zu entwickeln. So wird in dieser Phase eine Minimalversorgung aufrechterhalten [11,12]. Dieser als 


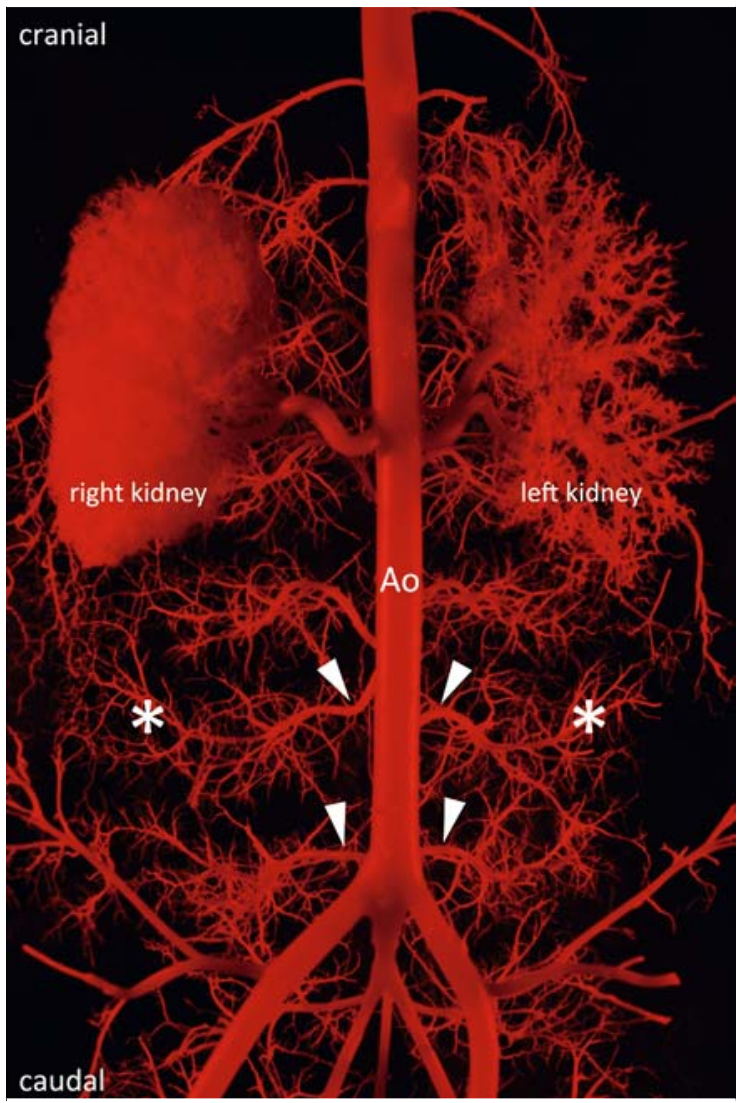

Abb. 1 Darstellung des reichhaltigen paraspinalen Kollateralnetzwerks als Gefäßausguss der abdominalen Aorta, der Nierenarterien, der Nieren und der dorsalen Segmentarterien im Schwein. Abgänge der proximalen Segmentarterien (weisse Pfeilspitzen) mit 2-3 Abgängen zum intraspinalen Netzwerk und der ausgeprägten Anastomosen zum paraspinalen Kollateralnetzwerk (weiße*), Ao $=$ Aorta.

„(Prä-)Konditionierung“ bezeichnete Umbauprozess im Kollateralnetzwerk unterliegt einer zeitlichen Latenz zwischen der teilweisen und der kompletten Segmentarterienopferung. Diese tritt abrupt während der offen-chirurgischen und der endovaskulären Therapie der thorakoabdominellen Aorta ein.

Basierend auf diesen Erkenntnissen entwickelten Etz und Griepp das gestufte Therapieverfahren (Staged Repair) als Konzept für die Versorgung von thorakoabdominellen Aortenerkrankungen [13]. Patienten mit folgender Operationssequenz hatten eine bis zu 10-fach niedrigere Paraplegierate [14]:

- In der 1. Operation wurde bereits ein Teil der Segmentarterien geopfert (z. B. nach Ersatz der Aorta thoracalis descendens).

- In der 2. Operation fand ein Ersatz der Aorta abdominalis statt.

Haulons Gruppe konnte erstmals in einer großen klinischen Serie eine erhebliche Senkung des Paraplegierisi- kos von 14,0 auf 1,2\% zeigen (allerdings in einer sehr heterogenen Patientenkohorte von Crawford Typ I bis III) [14]. Dies entspricht ebenfalls einer Senkung um den Faktor 10.

\section{Neuromonitoring während Aorteneingriffen}

\section{Messung motorisch und somatosensibel evozierter Potenziale}

Vielerorts wird ein klinisches Neuromonitoring unter Zuhilfenahme von MEP (motorisch evozierten Potenzialen) und SSEP (somatosensibel evozierten Potenzialen) während Eingriffen an der thorakoabdominellen Aorta routinemäßig angewendet [15]. Es dient dazu, frühzeitig Funktionsausfälle (direkt) bzw. Perfusionsdefizite des Rückenmarkgewebes (indirekt) zu erkennen. Diese Methodik zur intraoperativen Überwachung der Rückenmarkfunktion ist jedoch aufwendig (invasiv) und bedarf eines nicht unerheblichen technischen Aufwands. Monitoring von MEP und SSEP gibt Auskunft über die Funktionalität des Rückenmarks. Ein entscheidender Nachteil der Methode ist allerdings die weitestgehend unklare zeitliche Latenz und Ausprägung, mit der eine Signalreduktion als Surrogat beginnender Funktionseinschränkung eintritt.

\section{Nahinfrarotspektroskopie des Kollateralnetzwerks}

Eine neue Methode zum nicht invasiven Echtzeit-Monitoring der Rückenmarkoxygenierung stellt die indirekte cnNIRS (Kollateralnetzwerk-Nahinfrarotspektroskopie) dar. Dieses Verfahren misst die Oxygenierung im paraspinalen Kollateralnetzwerk ( $>$ Abb. 2). Diese ist, basierend auf dem Konzept des Kollateralnetzwerks, direkt proportional zur Oxygenierung im regionalen Rückenmarkgewebe [16]. Erste experimentelle und klinische Studien dieses Verfahrens sind vielversprechend [1720]. Es bedarf jedoch noch weiterer Untersuchungen, insbesondere hinsichtlich Referenzbereichen und klinischen Korrelationsanalysen. Erst dann kann dieses neue Verfahren generell empfohlen werden. Des Weiteren ist seine Anwendbarkeit bei der endovaskulären Therapie bisher noch nicht ausreichend untersucht.

\section{Klinisch-neurologische Evaluation}

Intraoperativ, bei TEVAR in Lokalanästhesie und generell während des postoperativen Verlaufs am wachen Patienten stellt nach wie vor die engmaschige klinischneurologische Evaluation einen integralen Bestandteil des neurologischen Monitoring dar.

Die klinisch häufigste Präsentation des postoperativen ischämischen Rückenmarkschadens ist das sog. A.-spinalis-anterior-Syndrom. Dies hat aufgrund der hypoxischen Schädigung der Vorderhörner und des Tractus 


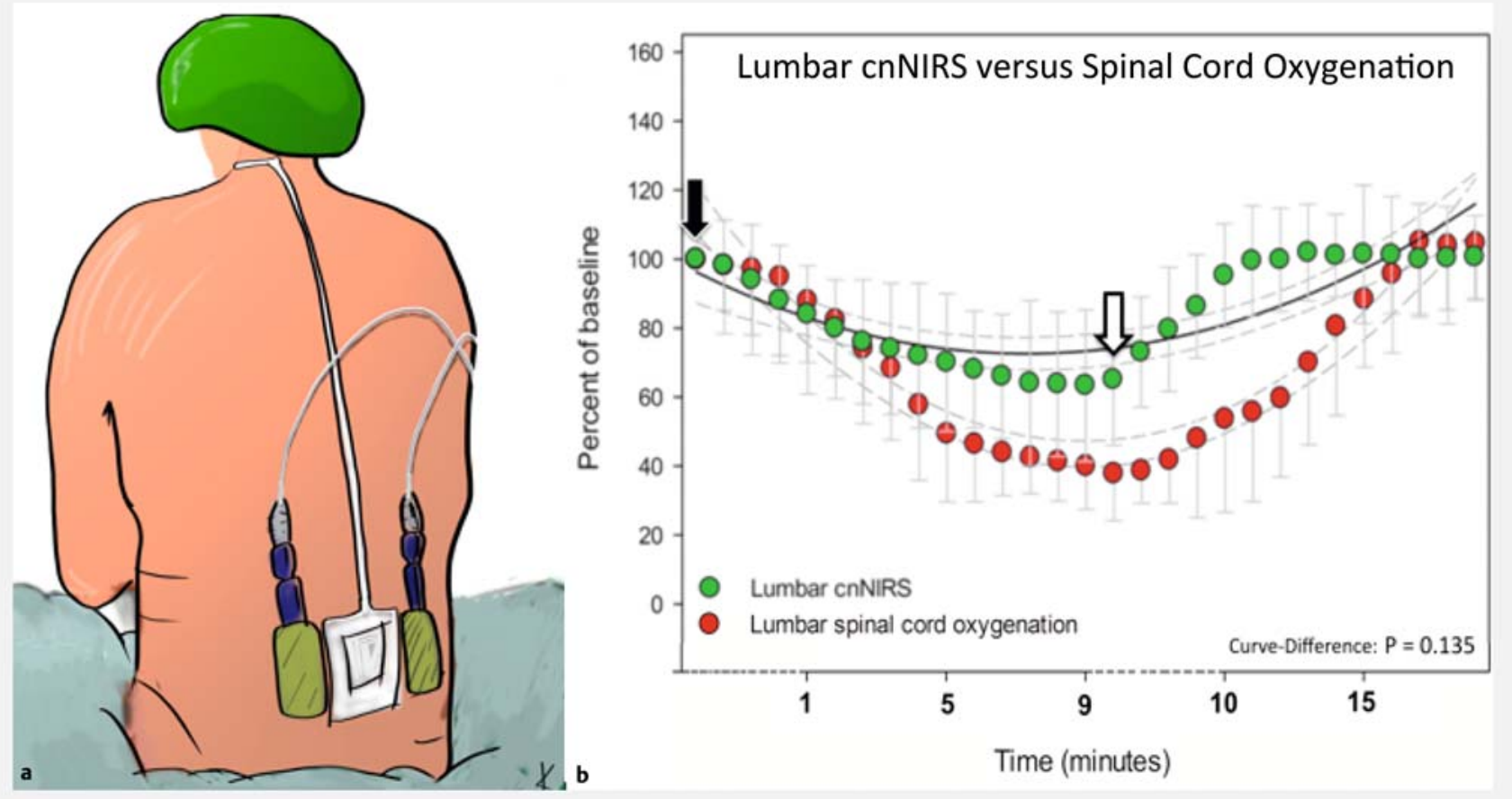

- Abb. 2 Lumbale cnNIRS. a Setup der lumbalen cnNIRS zur regionalen Oxygenierungsmessung der paraspinalen Muskulatur. b Vergleich der lumbalen cnNIRS mit direkter Rückenmarkoxygenierung nach Aortenklemmung und während Reperfusion.

spinothalamicus eine motorische Schwäche beider Beine bis hin zur Paraplegie zur Folge. Zudem kommt es zu einer dissoziierten Empfindungsstörung mit isoliertem Ausfall der Schmerz- und Temperaturempfindung bei weitestgehend erhaltener Sensibilität. Es empfiehlt sich daher generell, eine fachärztliche neurologische Untersuchung durchzuführen. Im Falle einer diagnostizierten Funktionsstörung ist auch eine weiterführende bildgebende Untersuchung mittels MRT (Magnetresonanztomografie) vom Rückenmark indiziert. Leider sind auch dann die Therapieoptionen begrenzt, nicht zuletzt aufgrund der extrem kurzen Ischämietoleranz des Rückenmarks.

\section{Merke}

Die frühzeitige Erkennung des verzögerten ischämischen Rückenmarkschadens (orientierend auch durch nicht ärztliches Personal) ist besonders wichtig. Nur so können standardisierte Gegenmaßnahmen (s. Abb. 3) ohne zeitlichen Verlust eingeleitet werden.

\section{Konventionelle Therapiemaßnah- men zur Rückenmarkprotektion}

\begin{abstract}
Merke
Das konventionelle Management während und nach komplexen Rekonstruktionen der thorakoabdominellen Aorta zielt darauf ab, eine möglichst suffiziente Perfusion und Oxygenierung des Rückenmarks zu gewährleisten.
\end{abstract}

\section{Blutdruckmanagement}

Eine zentrale Rolle in der Rückenmarkprotektion spielt der postoperative arterielle Blutdruck des Patienten. Dabei ist weniger der absolute Blutdruck, sondern vielmehr der Blutdruck relativ zum individuellen Ausgangswert des Patienten entscheidend [12].

Der postoperativ (auf der Intensivstation) eingestellte arterielle Blutdruck ist ausschlaggebend: Patienten mit postoperativen Blutdruckwerten entsprechend der individuellen Ausgangswerte oder oberhalb davon haben eine signifikant bessere Prognose hinsichtlich ischämischer Rückenmarkschäden [12].

Aktuelle Untersuchungen deuten ebenfalls darauf hin, dass insbesondere der systolische Blutdruck als Richtwert eine größere Rolle als der arterielle Mitteldruck 


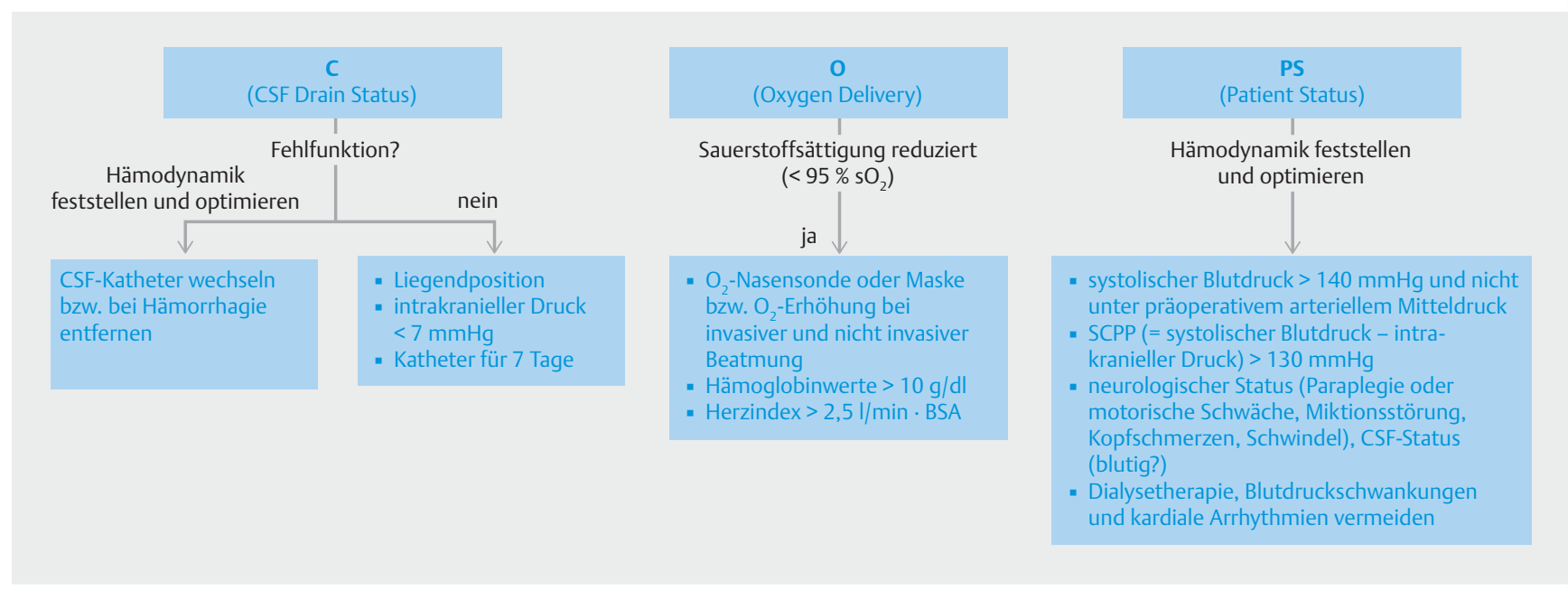

- Abb. 3 COPS-Schema - Notfallplan bei neurologischem Defizit. Modifiziertes Schema in Anlehnung an [33]. BSA=Körperoberfläche (Body Surface Area); $C S F=$ Liquor cerebrospinalis (Cerebrospinal Fluid); $\mathrm{O}_{2}=$ Sauerstoff; SCPP= Rückenmarkperfusionsdruck (Spinal Cord Perfusion Pressure)

spielt. Er sollte zumindest bei Werten oberhalb von 140 $\mathrm{mmHg}$ gehalten werden [21]. Die Empfehlung geht dahin, ein aggressives Management zur Substitution von Sauerstoffträgern durchzuführen, um die regionale Perfusion und Oxygenierung zu optimieren. Als Zielparameter wird ein Hämoglobingehalt von über $10 \mathrm{~g} / \mathrm{dl}$ angestrebt. Die insbesondere nach offenem Ersatz der thorakoabdominellen Aorta notwendige Volumentherapie birgt jedoch das Risiko eines Anstiegs des zentralen Venendrucks. Die Folge ist die indirekte Erhöhung des Druckes des Liquor cerebrospinalis. Deshalb sollte der intravenösen Gabe von Vasopressoren in dieser Situation der Vorzug gegeben werden [12].

Der Rückenmarkperfusionsdruck kann mittels folgender Formel grob eingeschätzt werden:

$$
\begin{gathered}
\mathrm{SCCP}=\mathrm{MAP}-\mathrm{CSF}-\text { Druck } \\
\text { mit } \\
\mathrm{SCCP}=\mathrm{Rückenmarkperfusionsdruck} \\
\mathrm{MAP}=\text { arterieller Mitteldruck }
\end{gathered}
$$

CSF-Druck = Druck des Liquor cerebrospinalis (als Surrogat für den CSF-Druck kann annähernd auch der zentrale Venendruck herangezogen werden)

\section{Liquordrainage}

Der effektive Perfusionsdruck im Rückenmark ist nicht nur vom systemischen arteriellen Druck, sondern auch vom intraspinalen Druck abhängig. Deshalb wird eine konsequente Liquordrainage zur Senkung des intrakraniellen Druckes auf Werte unter $7 \mathrm{mmHg}$ empfohlen. Diese Maßnahme wird in vielen Kliniken routinemäßig angewendet $[3,22,23]$.

\section{Cave}

Akute Senkungen des Druckes des Liquor cerebrospinalis durch exzessive Drainage können mit einem nicht unerheblichen Risiko einer Brückenvenenblutung einhergehen und müssen unbedingt vermieden werden [24].

Zurzeit existieren keine kontrollierten, randomisierten Studien hinsichtlich Liquordrainage in der Therapie von thorakoabdominellen Aortenerkrankungen. Vorliegende aortenchirurgische Studien unterstützen jedoch die Anwendung dieser Methode zur Rückenmarkprotektion $[21,22,25]$. Die durch lumbale Liquordrainagen ausgelösten neurologischen Komplikationen sind mit einer Inzidenz von 0,8\% sehr selten [25]. Allgemeine Komplikationen wie z.B. blutig tingierter Liquor (10,0\% der Fälle) oder epidurale Hämatome einschließlich intrakranieller Blutungen (5,2\%) waren in der Regel asymptomatisch. Sie waren oft assoziiert mit einem er-

\section{PRAXIS/MAßNAHMEN}

Allgemeine Empfehlungen zur korrekten und sicheren Anwendung der lumbalen Liquordrainage beinhalten eine adäquate Gerinnungssituation bereits vor Katheteranlage:

- keine Thrombozytenaggregationshemmung außer mit Azetylsalizylsäure in Standarddosierung

- Thrombozytenzahlen von mindestens $100000 / \mu \mathrm{l}$

- INR-Wert (Wert der International normalized Ratio) von unterhalb 1,3 bei normaler partieller Thromboplastinzeit 
höhten zentralvenösen Druck und einer derangierten Gerinnung des Patienten [25].

Nach Anlage sollte eine Vollheparinisierung frühestens $1 \mathrm{~h}$ später erfolgen. Zum Erreichen des intrakraniellen (=spinalen) Zieldrucks sollte nach Möglichkeit das Drainieren von $15 \mathrm{ml}$ Liquor pro Stunde nicht überschritten werden [22]. Die manuelle Liquordrainage ist diesbezüglich gefährlicher als ein passives Drainieren (gegen Gravitation). Um die effektive Rückenmarkperfusion aufrechtzuerhalten, ist bei liegendem Liquor-cerebrospinalis-Katheter generell eine systemische Hypotension zu vermeiden. Es ist zum jetzigen Zeitpunkt noch unklar, inwieweit die aktiv-automatische Liquordrainage (LiquoGuard ${ }^{\circledR}$ ) einen Vorteil gegenüber Methoden der intermittierend manuellen oder passiv gegen die Schwerkraft drainierenden Techniken besitzt. Entsprechende klinische und experimentelle Studien laufen derzeit an. Das Entfernen der Drainage bei ausgeglichener Gerinnung wird nach $72 \mathrm{~h}$ postoperativ empfohlen. Die Drainage sollte jedoch im Falle neurologischer Komplikationen, die nicht im kausalen Zusammenhang mit der Drainage selbst stehen, bis zu 7 Tagen belassen werden. Dadurch lässt sich eine adäquate Senkung des intraspinalen Druckes gewährleisten [26].

\section{Antikoagulation}

Es konnte gezeigt werden, dass der thrombembolische Verschluss der rückenmarkversorgenden Gefäße ein möglicher und unterschätzter Mechanismus in der Entstehung des ischämischen Rückenmarkschaden ist [27]. Deshalb wird die therapeutische Gabe von Heparin für die ersten 3 Monate nach festgestelltem neurologischem Defizit empfohlen. Im Falle eines liegenden Spinalkatheters erfolgt die erste Gabe frühestens $1 \mathrm{~h}$ nach Entfernung [28]. Entsprechend der aktuellen Studienlage ist nicht zu beantworten, inwieweit dies auch prophylaktisch nach ausgedehnten Operationen an der thorakoabdominellen Aorta zu empfehlen ist [28]. Insbesondere nach offen-chirurgischem Aortenersatz führen Blutungskomplikationen nicht selten zu Hypotension und regionaler Hypoxämie. Darum wird neben der Gabe von Erythrozytenkonzentraten und Frischplasma auch die Substitution von Thrombozyten auf Werte oberhalb von $100 \quad 000 / \mu$ l empfohlen (ggf. Thrombozyten-Funktionstestung/TEG).

\section{Medikamentöse Rückenmarkprotektion}

\footnotetext{
Merke

Zum aktuellen Zeitpunkt existieren keine klinischen Studien, die einen Vorteil einer medikamentösen Rückenmarkprotektion bei Interventionen an der thorakoabdominellen Aorta in Bezug auf neurologische Defizite nachweisen.
}

In einigen experimentellen Studien ergaben sich für gewisse Substanzen jedoch positive Effekte auf die Rückenmarkperfusion [29]. Im Ischämie-/Reperfusionsexperiment am Großtier bewirkte die intrathekale Gabe von Papaverin eine signifikant bessere Rückenmarkperfusion. Auch die intravenöse Gabe von Substanzen wie Naloxon und Mannitol hatte experimentell positive Auswirkungen auf die Rückenmarkdurchblutung. Wenige spezialisierte Zentren verwenden sie deshalb bei Operationen an der thorakoabdominellen Aorta [28]. Sowohl für diese Substanzen als auch hinsichtlich der Anwendung von Kortikosteroiden zur Inflammationshemmung konnte kein Nutzen in der Prophylaxe oder Therapie des ischämischen Rückenmarkschadens nach Aortenoperationen nachgewiesen werden [28, 29]. Im Gegensatz dazu stehen Ergebnisse ihrer Anwendung bei der traumatischen Rückenmarkschädigung.

\section{Cave}

Die Gabe von Vasodilatatoren (z. B. von Nitraten und $\alpha$-Blockern) kann zu Hypotension durch arteriovenöse Shunts führen. Deshalb werden sie in der peri- und postoperativen Therapie von thorakoabdominellen Aortenerkrankungen nicht empfohlen [29].

\section{Lokale Rückenmarkkühlung}

In Anbetracht möglicher Risiken der intraoperativen systemischen Hypothermie wurde in einigen experimentellen Studien und vereinzelt auch bereits in klinischer Anwendung eine Methode der lokal begrenzten Hypothermie verwendet. Diese Methode wird als „Epidural Cooling“ bezeichnet [30]. Dabei wird gezielt der Bereich des Rückenmarks durch Hypothermie geschützt, der typischerweise dem Risiko einer ischämischen Schädigung während der operativen Versorgung eines thorakoabdominellen Aortenaneurysmas ausgesetzt ist. Die Kühlung erfolgt durch epidurale Kühlung mit $4{ }^{\circ} \mathrm{C}$ kalter Lösung. Die Ergebnisse erster klinischer Studien waren vielversprechend [31].

\footnotetext{
Merke

Der technische Aufwand und die Invasivität stellen wesentliche Limitationen der epiduralen Kühlung dar. Weitere Nachteile sind der Anstieg des intrakraniellen Druckes und der damit verbundene reduzierte spinale Blutfluss. Daher ist eine kontinuierliche Liquordrainage unerlässlich.
} 


\section{COPS-Schema}

PRAXIS/MABNAHMEN

Empfehlungen für die konventionelle Rückenmarkprotektion [32]:

- hochnormaler, individueller Blutdruck (mittlerer Blutdruck oberhalb des individuellen Ausgangswerts, systolischer Blutdruck zumindest über $140 \mathrm{mmHg}$ )

- lumbale Liquordrainage (intrakranieller Druck unter $7 \mathrm{mmHg}$; Drainage maximal $15 \mathrm{ml} / \mathrm{h}$ )

- Hämoglobinkonzentration über $10 \mathrm{~g} / \mathrm{dl}$ und Thrombozytenzahl über $100000 / \mu \mathrm{l}$

- Sauerstoffsättigung über $95 \%$

- keine Gabe von Nitraten oder $\alpha$-Blockern

- cave bei Volumentherapie (Vasopressoren bevorzugen)

Das COPS-Protokoll subsumiert alle genannten Therapiekonzepte für die frühe postoperative Phase, während der das Risiko der verspäteten Spinalischämie am größten ist. Diese Phase liegt zwischen dem 2. und dem 5. postoperativen Tag [26]. Für diesen Fall empfiehlt sich die unmittelbare und konsequente Anwendung des modifizierten COPS-Konzepts ( $>$ Abb.3) [26, 33]. Das Konzept basiert auf aktuellen Erkenntnissen auf dem Gebiet der Rückenmarkprotektion im Zusammenhang mit Eingriffen an der thorakoabdominellen Aorta.

\section{Rückenmarkprotektion bei offen- chirurgischem Aortenersatz}

\section{Temperaturmanagement}

Speziell während des offen-chirurgischen Aortenersatzes ist vor allem ein adäquates Temperaturmanagement ein wichtiger Aspekt zur Vorbeugung eines intraoperativen ischämischen Rückenmarkschadens während der Abklemmung der Aorta (Cross-Clamping). Nachweislich ist die Inzidenz postoperativer Organschäden bei Operationen unter tiefer und profunder Hypothermie geringer, verglichen mit milder Hypothermie [34]. Die systemische Hypothermie als effektive Methode der Organprotektion ist jedoch in Abhängigkeit der Minimaltemperatur mit unerwünschten Risiken verbunden: Neben Herzrhythmusstörungen und einer Verminderung der kardiopulmonalen Funktion werden häufig erworbene Koagulopathien diskutiert [30]. Die meisten Zentren wenden daher ein Regime der moderaten Hypothermie von ca. $32{ }^{\circ} \mathrm{C}$ an [29]. Einige Studien konnten jedoch zeigen, dass sich die Rate an blutungsassoziierten Komplikationen bei Patienten operiert unter tiefer Hypothermie im Vergleich zur milden Hypothermie nicht signifikant unterscheidet. Viel- mehr ist ein Zusammenhang mit der unter extrakorporaler Zirkulation oftmals notwendigen Vollheparinisierung anzunehmen [34]. Für das frühe postoperative Management haben hochspezialisierte Zentren Protokolle für Patienten nach offenem Aortenersatz unter tiefer oder moderater Hypothermie entwickelt. Diese beinhalten u.a., dass Patienten über mehrere Stunden auf der Intensivstation bei Raumtemperatur nur passiv gewärmt werden [29].

\section{Serielles Abklemmen der Aorta}

Eine zunehmend angewandte Technik beim offenen Ersatz der thorakoabdominellen Aorta stellt das serielle Cross-Clamping dar. Durch sequenzielles Umsetzen der Aortenklemme von proximal nach kaudal wird eine frühzeitige Freigabe der direkten Blutversorgung durch (reimplantierte) Segmentarterien erreicht und so das Risiko des intraoperativen Rückenmarkschadens reduziert [35].

\section{Distale Aorten- und selektive Organperfusion}

Merke

Vielleicht den wichtigsten Aspekt für die intraoperative Rückenmarkprotektion stellt die distale Perfusion mittels extrakorporaler Zirkulation (mit oder ohne Oxygenator) dar.

Obwohl keine randomisierten Studien die Effektivität der distalen Perfusion hinsichtlich des neurologischen Outcome untersucht haben, geht diese mit dem Konzept des Kollateralnetzwerks Hand in Hand. Denn die kontinuierliche distale Perfusion in das Kollateralnetzwerk wird intraoperativ gewährleistet. Daten der cnNIRS korrelieren signifikant mit dem Einsatz distaler Perfusion [16]. Wir sehen distale Perfusion daher als essenziell für den komplexen, langstreckigen Ersatz der thorakoabdominellen Aorta [3].

\section{Reimplantation aortaler Segmentarterien}

Die Rolle der Reimplantation von aortalen Segmentarterien ist trotz langjähriger Forschung und klinischer Erfahrung noch nicht ausreichend verstanden.

Aktuelle Studien und Metaanalysen zeigen keine wesentlichen Vorteile der Reimplantation in Bezug auf das neurologische Outcome [6]. Vielmehr hatte die Anwendung konventioneller, auf den neuen Erkenntnissen der Rückenmarkperfusion beruhender Konzepte einen größeren Einfluss auf ein positives neurologisches Ergebnis [6]. Es konnte also kein eindeutiger Vorteil der selektiven Reimplantation der Segmentarterien nachgewiesen werden. Trotzdem ist sie nach wie vor Gegenstand von Diskussionen unter Experten und gehört in vielen Aortenzentren zur Standardtherapie. 
Ein möglicher Vorteil der Reimplantation ist die frühe Wiederherstellung einer direkten Perfusion des Rückenmarks über die Segmentarterien (vgl. die sequenzielle Aortenklemmung). Es zeigen sich jedoch unsichere Offenheitsraten im Verlauf [36]. Vorteile einer raschen Okklusion der Segmentarterien:

- Ausbleiben von Rückblutungen (sog. Steal aus dem Kollateralnetzwerk), dadurch

- allgemein übersichtlicheres Operationsfeld bei kürzerer Cross-Clamp-Zeit

Diese Vorteile fallen weg bei einem bereits präoperativen iatrogenen Verschluss (MISACE [Minimally invasive segmental Artery Coil and Plug Embolization]; s. unten). Basierend auf Erkenntnissen des modernen Konzepts der Rückenmarkperfusion ist die Strategie der Reimplantation der Segmentarterien aktuell jedoch im Mittelpunkt der Expertendiskussion [4,6]. Ein Paradigmenwechsel scheint deshalb wahrscheinlich.

\section{Rückenmarkprotektion bei endovaskulärer Therapie}

Einige spezialisierte Zentren haben optimierte Protokolle entwickelt und implementiert, um dem erhöhten Risiko einer spinalen Ischämie durch die endovaskuläre Therapie (TEVAR) ausgedehnter thorakoabdomineller Aortenerkrankungen (Crawford-Typ II) entgegenzuwirken.

\section{Etablierte Methoden}

Analog zu den etablierten Methoden des offen-chirurgischen Aortenersatzes (s. oben) wird auch für TEVAR ein hochnormaler Blutdruck angestrebt. Zusätzlich konnte gezeigt werden, dass die Strategie eines frühzeitigen Entfernens der femoralen Schleuse aus der Beckenachse einen positiven Effekt auf das neurologische Outcome hat [14]. Diese Maßnahme ist mit Perfusionsfreigabe über die Aa. iliacae in das rückenmarkversorgende paraspinale Kollateralnetzwerk verbunden (vgl. distale Perfusion). Die gestufte Therapie (Staged Re-

PRAXIS/MABNAHMEN

Die gestufte Therapie (Staged Repair) hat eine entscheidende Rolle in der modernen Rückenmarkprotektion bei endovaskulären Eingriffen an der Aorta thorakoabdominalis. Es ist sinnvoll, zur Rückenmarkprotektion bei endovaskulärer Therapie kleinkalibrige Schleusen zu verwenden und die Perfusion über die A. subclavia sinistra und die A. mesenterica inferior zu erhalten, um den Zustrom zum Kollateralnetzwerk aufrechtzuerhalten. pair) scheint eine entscheidende Bedeutung hinsichtlich des neurologischen Outcome zu haben. Diese wurde bereits 2009 von Etz und Mitarb. vorgeschlagen und ist mittlerweile auch in der endovaskulären Therapie im Begriff, etabliert zu werden. Ein aggressives Management hinsichtlich Erythrozyten- und Thrombozytentransfusion und die kontinuierliche Liquordrainage hatten ebenfalls einen positiven Effekt auf das postoperative neurologische Outcome [14]. Die prophylaktische Anlage einer Liquordrainage wird in der Regel jedoch erst dann empfohlen, wenn der Stentgraft die Aorta auf einer Strecke von mehr als $12 \mathrm{~cm}$ bedeckt [21].

Generell werden auch für die endovaskuläre Therapie die gleichen Prinzipien wie bei der konventionellen Rückenmarkprotektion angewendet und kombiniert, um das Risiko der peri- und postoperativen Rückenmarkischämie möglichst gering zu halten.

\section{Vorübergehende Aneurysmaperfusion}

Ein relativ neues und noch selten verwendetes Verfahren zur möglichen Prophylaxe eines ischämischen Rückenmarkschadens bei TEVAR stellt die vorübergehende Aneurysmaperfusion über dedizierte Abgänge der Stent-Prothese dar. Dieses Verfahren wird als „TASP“ bezeichnet (Temporary Aneurysm Sac Perfusion). Die Abgänge der Stent-Prothese können dann im Intervall endovaskulär wieder verschlossen werden. Die Perfusionsabgänge versorgen so einen Teil des Aneurysmas, bevor sie in einer 2. Sitzung (bis zu 4 Wochen später) verschlossen werden. Damit ist das Aneurysma komplett ausgeschaltet. Das ahmt praktisch einen Staged Repair nach.

Erste klinische Serien von TASP waren vielversprechend und zeigten eine signifikante Senkung der Rate von postoperativen neurologischen Komplikationen [37]. Aufgrund der anhaltenden Perfusion des Aneurysmas bis zur vollständigen Ausschaltung kann es jedoch in 26 \% der Fälle zur Progression des Aneurysmas kommen [37]. Ein gefürchtetes Risiko des Verfahrens ist die Embolisation distaler rückenmarkversorgender Arterien durch Translokation von Thromben und anderen Ablagerungen aus dem Aneurysmalumen. Zudem bleibt das Risiko einer spontanen Komplikation im Intervall bestehen. Klarer Nachteil dieser Methode ist damit die unvollendete Therapie des Aneurysmas, solange der Aneurysmasack unter Druck steht.

\section{Minimalinvasive Präkonditionierung des Kollateralnetzwerks}

Trotz aller Bemühungen zur Reduktion des interventionsbedingten ischämischen Rückenmarkschadens handelt es sich bei den konventionellen Strategien lediglich um unterstützende, adjunktive Maßnahmen 
und nicht um definitive, kausale Präventionskonzepte. Als neues Therapiekonzept mit kausalem Ansatz zur Rückenmarkprotektion wurde kürzlich die minimalinvasive endovaskuläre Präkonditionierung von Etz und Mitarb. eingeführt.

Das mehrzeitig gestufte Vorgehen (sog. Staged Repair) bildet dafür die klinische Grundlage. Es ermöglicht eine präoperative Konditionierung des paraspinalen Kollateralnetzwerks durch „Minimalinvasive Segmentarterien Coil- und Plug Embolization“ (MISACE) als Vorbereitung zum elektiven Ersatz der thorakoabdominellen Aorta

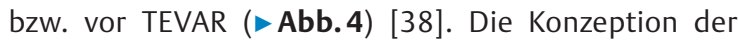
minimalinvasiven Präkonditionierung und ihre erste Anwendung am Menschen erfolgten 2013 durch Etz u. Kölbel [38]. MISACE wird in der Regel als Mehrfachintervention durchgeführt, um dem Kollateralnetzwerk zwischen den MISACE-Sitzungen ausreichend Zeit für die entsprechenden Umbauprozesse zu geben [38]. Das dazu notwendige Zeitintervall ist jedoch bislang nicht klar definiert. Bei ausgedehnten Aortenaneurysmen sind einige Segmentarterien bereits pathologisch verschlossen (z. B. bei chronischen Typ-B-Dissektionen). Deshalb ist in manchen Fällen nur eine einzige MISACEProzedur notwendig.

\section{Präprozedurale Diagnostik}

Die Diagnostik vor MISACE entspricht weitestgehend der für den elektiven Ersatz der thorakoabdominellen Aorta oder TEVAR. Einige prozedurspezifische Besonderheiten sollten jedoch beachtet werden.

\section{Merke}

Insbesondere die adäquate Darstellung der anatomischen Gegebenheiten mittels Kontrastmittel und Dünnschicht-CT (Dünnschichtcomputertomografie; $1 \mathrm{~mm}$ Schichtdicke oder dünner) ist vor MISACE essenziell.

Eine sagittale Darstellung der paarigen Segmentarterien in Bezug auf die Nierenarterien und andere größere Abdominalgefäße erleichtert die Orientierung während der folgenden Prozedur ( A Abb.5). Insbesondere bei Aneurysmen auf dem Boden chronischer TypB-Dissektion ist eine präinterventionelle Darstellung der Segmentarterien mit Bezug auf das wahre und falsche Lumen wichtig. So kann entsprechend die jeweils

\section{PRAXIS/MABNAHMEN}

Pro MISACE-Sitzung werden maximal 6- 7 Segmentarterien verschlossen. Während der Prozedur regelmäßige klinische Abfrage bezüglich Schmerzen und/ oder anderer neurologischer Symptome.

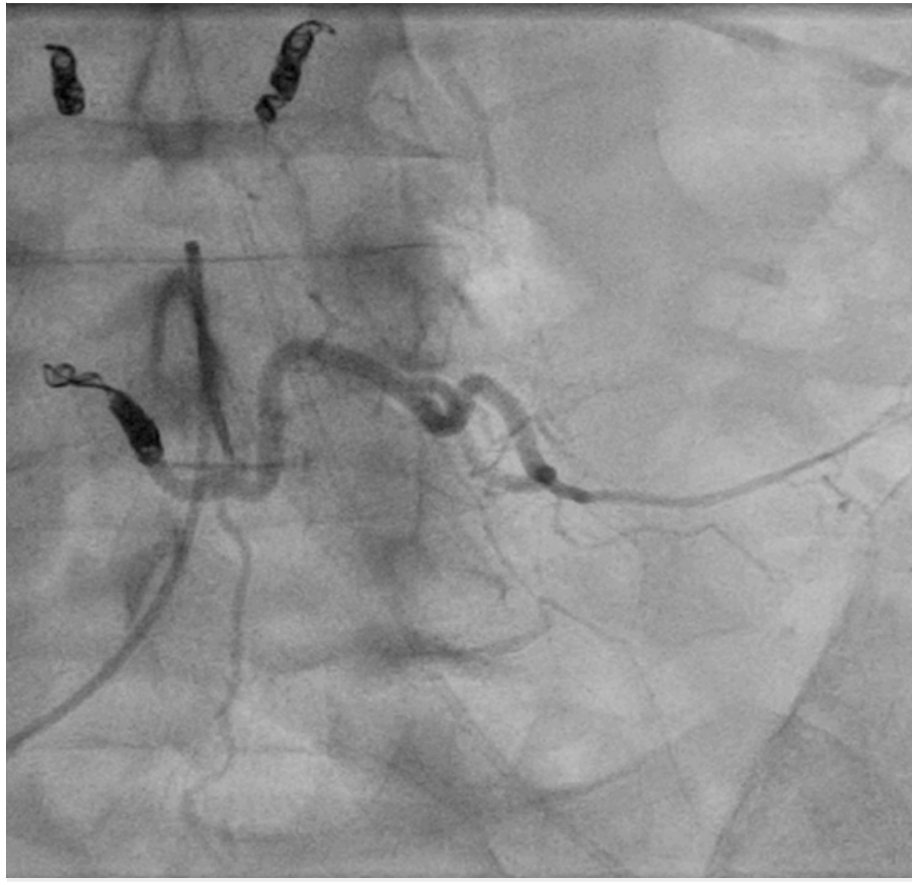

Abb. 4 MISACE. Angiografie einer MISACE-Prozedur mit Kontrastdarstellung einer offenen Segmentarterie und mehrerer Coils in insgesamt 3 weiteren Segmentarterien.

korrekte Seite zur Femoralispunktion gewählt werden. Bei Vorliegen aktueller und qualitativ hochwertiger CTBilder kann auf eine intraprozedurale angiografische Übersichtsaufnahme unter Umständen verzichtet werden.

\section{Absolute und relative Kontraindikationen}

Aufgrund der Neuartigkeit der Methode basieren Empfehlungen hinsichtlich Kontraindikationen weitestgehend auf Erfahrungswerten und Einschätzungen von Experten:

- absolute Kontraindikationen:

- Symptomatik (neue progrediente Rückenschmerzen, Durchblutungsstörung, thrombembolische Komplikationen)

- Aneurysmawachstum von über $1,5 \mathrm{~cm} / \mathrm{Jahr}$ bei bereits kritischem Aortendiameter (über $8 \mathrm{~cm}$ )

- mykotische Aortenaneurysmen (auch der Verdacht)

- frische Thrombenformation im Bereich Th12-L5 (sog. Shaggy Aorta; > Abb. 6 )

- relative Kontraindikationen:

- ausgeprägtes Kinking der Aorta (einschließlich der Beckengefäße) und/oder schwere Verkalkungen

- Vorhandensein von nur wenigen, kaliberschwachen, weniger als 4 oder keinen patenten Segmentarterien 

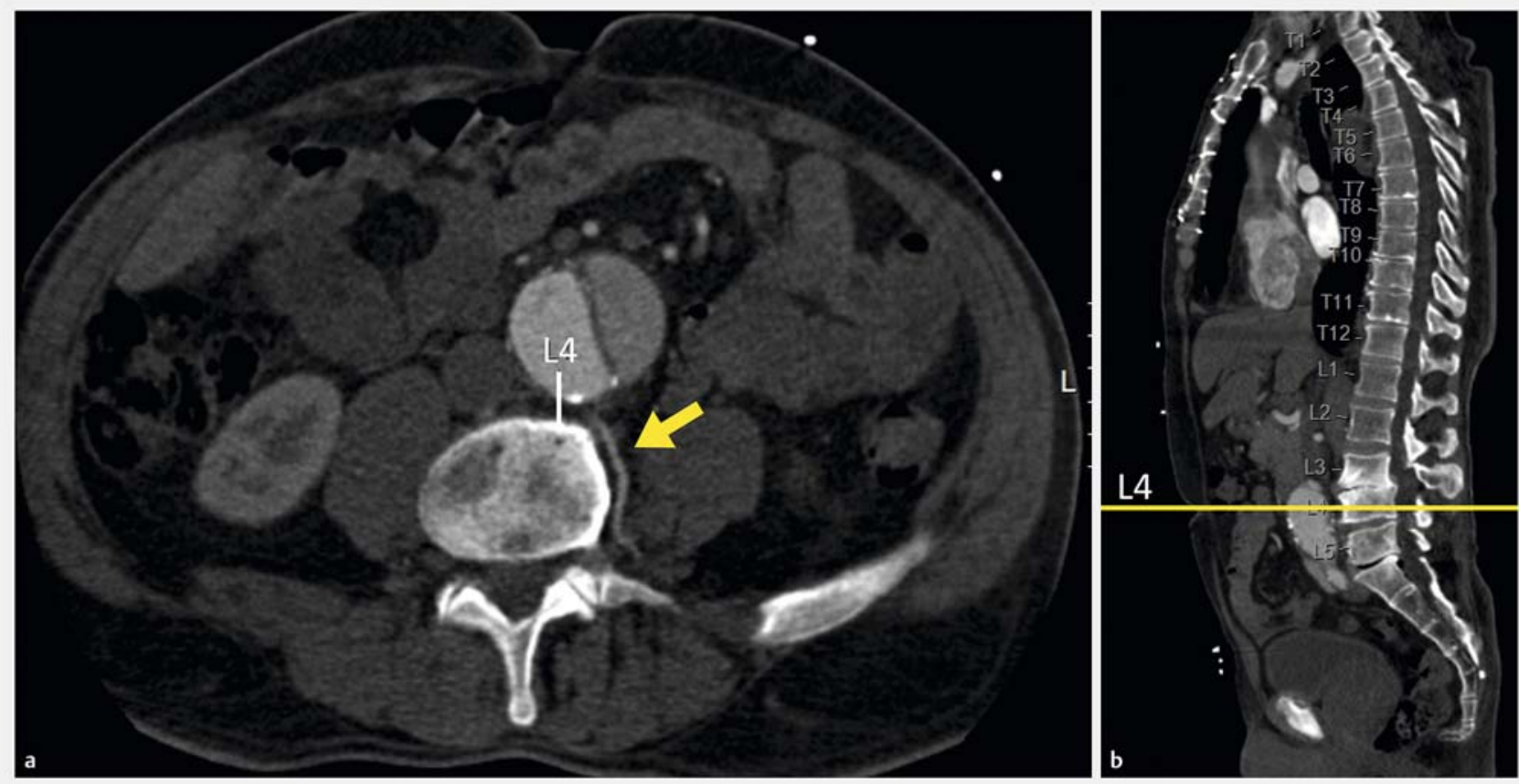

Abb. 5 Präoperative CT zur Darstellung der Segmentarterien mit Kontrastmittelangiografie. a Querschnitt auf Segmentebene L4 und Darstellung der linken Segmentarterie (Pfeil). b Sagittalschnitt mit Wirbelkörpermarkierung.
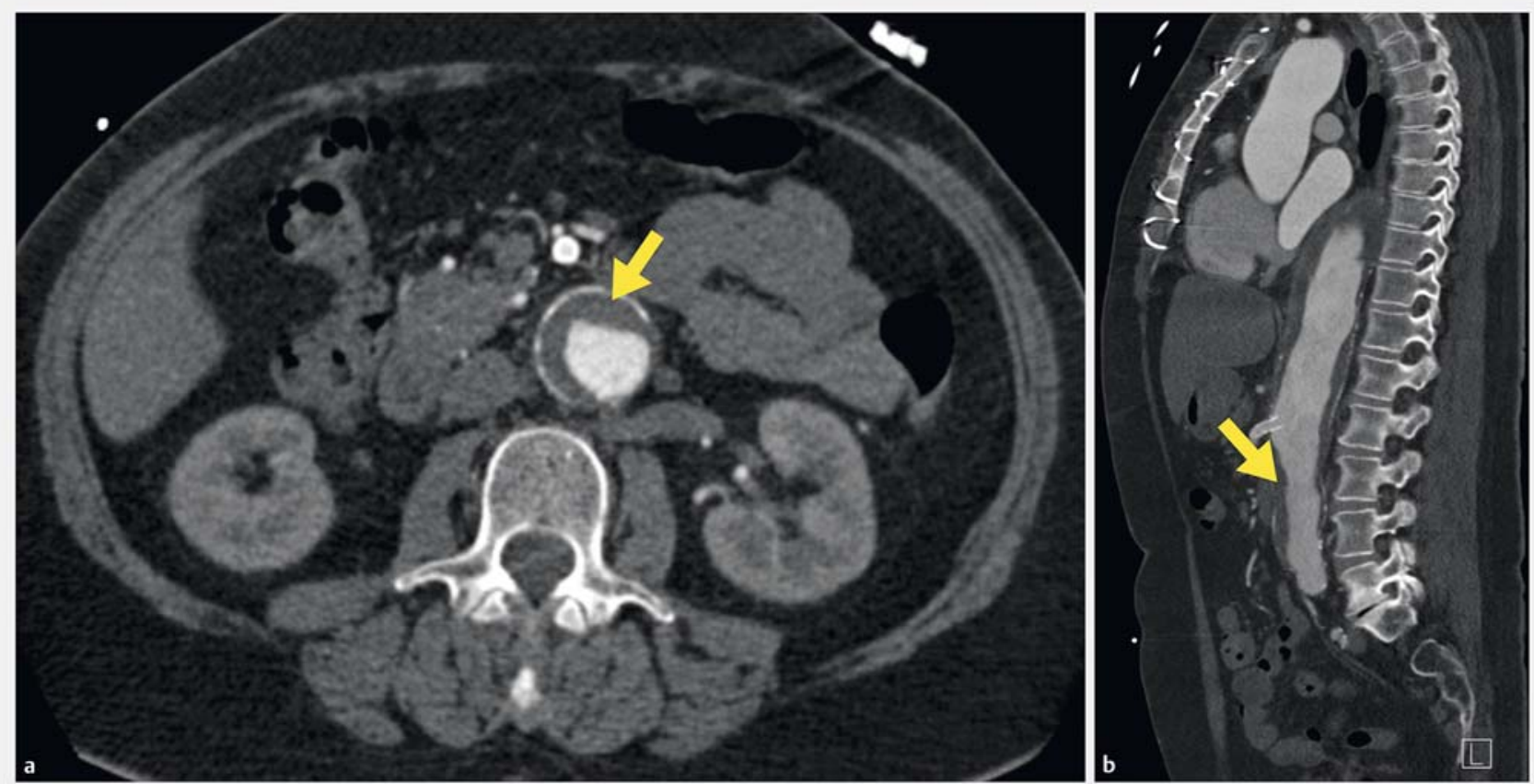

- Abb.6 Teilthrombosiertes thorakoabdominelles Aortenaneurysma mit pathologieassoziiertem Verschluss der Segmentarterien. a Querschnitt mit Darstellung des wandständigen Thrombus in der Aorta abdominalis (Pfeil) und pathologieassoziiertem Verschluss der Segmentarterien. b Sagittalschnitt des teilthrombosierten thorakoabdominellen Aortenaneurysmas. Der Pfeil kennzeichnet den Thrombus. 

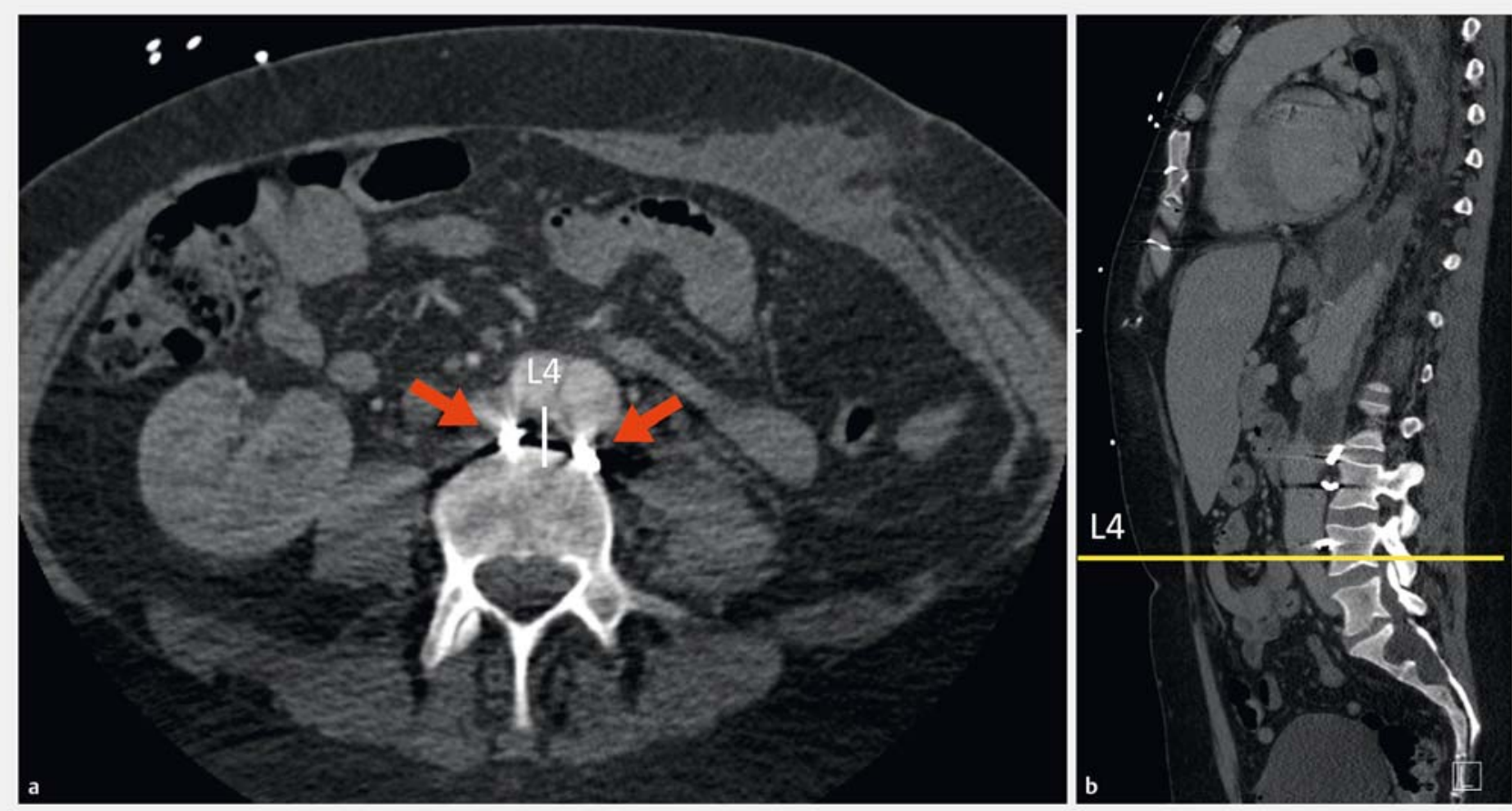

Abb. 7 CT nach der ersten MISACE-Sitzung. Dargestellt sind die beidseits embolisierten Segmentarterien L4 mittels Coils (Pfeile). a Querschnittaufnahme. b Sagittalaufnahme.

\section{Ablauf der Prozedur}

Nach Ausschluss relevanter Kontraindikationen findet die erste MISACE-Sitzung statt, die in Rückenlage durchgeführt wird. Der Zugang erfolgt nach örtlicher Betäubung über eine der beiden Aa. femorales. Meist wird die A. femoralis dextra gewählt. Jedoch ist dies abhängig von den anatomischen Gegebenheiten, wie z. B. einem Kinking der Beckenachse oder der Lokalisation des wahren bzw. falschen Lumens in Bezug auf die linke oder rechte Beckenarterie. Durch eine 4- oder 5-FSchleuse wird der Pigtail-Katheter in die Aorta vorgeschoben. In einigen Fällen wird eine Aortenübersichtsaufnahme angefertigt. Dies sollte aber insbesondere bei eingeschränkter Nierenfunktion vermieden werden. Die im Team präoperativ avisierten Segmentarterien werden selektiv aufgesucht und mittels Kontrastmittel dargestellt. Die jeweilige Arterie wird mit einem Stabilisierungskatheter (Mikrokatheter) intubiert und mit einem entsprechend dimensionierten Coil (oder Vascular Plug) verschlossen ( $\bullet$ Abb. 7). In der Regel sind mehrere Coils pro Gefäß notwendig. Es wird darauf geachtet, die Coils im Anfangsbereich der Segmentarterie zu positionieren und nicht nach distal zu dislozieren.

Bei Symptomen (z. B. dumpfen tiefen Rücken- oder Gesäßschmerzen, Tachykardie, Hypotonie oder neurologischen Defiziten) muss der unmittelbare Abbruch der
Prozedur erwogen werden. Insbesondere Schmerzen im tiefen Rücken- oder Gesäßbereich deuten auf eine relative Ischämie hin. Diese ist jedoch erfahrungsgemäß nur temporär und verschwindet bei adäquatem hochnormalem Blutdruckmanagement innerhalb weniger Stunden.

Ein wichtiger Aspekt von MISACE stellt der adäquate Verschluss der zu embolisierenden Gefäße dar. Prinzipiell - aber insbesondere wenn Coils mit erhöhter Thrombogenität verwendet werden - sollten ausreichend Coils/ bzw. Vascular Plugs in das Zielgefäß eingebracht werden, sodass der Fluss distal möglichst zeitnah unterbunden ist (Kontrolle mittels KM Applikation bei noch liegendem Mikrokatheter), um das Risiko einer distalen Embolisation durch thrombotisches Material aus dem Okklusionsbereich zu reduzieren.

Es ist hilfreich, während der ersten Sitzung bereits Besonderheiten in Hinblick auf eine mögliche 2. Sitzung zu vermerken, z.B. bezüglich nicht patenter bzw. schwer darstellbarer Segmentarterien, einer optimalen Katheter- bzw. Zugangsarterie.

Nach Abschluss der Prozedur wird der Patient für $72 \mathrm{~h}$ auf einer Monitorstation mit invasiver Blutdruckmessung weiter klinisch, hämodynamisch und neurologisch überwacht. Der Blutdruck ist entsprechend der konventionellen Rückenmarkprotektion für die ersten 


\section{PRAXIS/MAßNAHMEN}

Insbesondere bei der Coil-Embolisation der A. mesenterica inferior (zur Endoleak-Prävention vor TEVAR), ist darauf zu achten, dass keine unterdimensionierten Coils verwendet werden. Denn es besteht die Gefahr einer distalen Translokation mit konsekutivem Verschluss der A. colica sinistra (Riolan-Anastomose). Es empfiehlt sich daher die Verwendung eines Vascular Plug zur Okklusion.

5 Tage auf einem Niveau über den individuellen Ausgangswerten zu halten. Auf Vasodilatatoren (insbesondere Nitrate) sollte während dieser Zeit ebenfalls verzichtet werden.

Im Aorten-Team muss anschließend entschieden werden, ob eine Wiederaufnahme zu einer 2. MISACE-Sitzung oder zur definitiven Operation im Intervall erfolgt. Das empfohlene Intervall liegt derzeit bei 3-4 Wochen. Bei Symptomatik während dieser Zeit ist eine sofortige Wiederaufnahme und Operationsplanung notwendig.

\section{Besonderheiten bei endovaskulärer Therapie}

MISACE findet nicht nur zur Präkonditionierung des Kollateralnetzwerks vor geplanten Eingriffen an der thorakoabdominellen Aorta Anwendung, sondern ebenfalls bei Patienten vor geplantem infrarenalem

\section{KERNAUSSAGEN}

- Sowohl beim chirurgischen Ersatz der thorakoabdominellen Aorta als auch bei TEVAR im Rahmen der Aneurysmatherapie ist das Risiko der ischämischen Rückenmarkschädigung nach wie vor sehr hoch.

- Mithilfe eines adäquaten peri- und postoperativen Managements, basierend auf dem modernen Konzept der Rückenmarkperfusion, kann dieses Risiko signifikant gesenkt, jedoch bisher nicht eliminiert werden.

- Die von Etz und Mitarb. kürzlich eingeführte MISACE ist ein katheterbasiertes Verfahren zur Rückenmarkprotektion durch präoperative Konditionierung, das in jedem Katheterlabor unproblematisch durchgeführt werden kann.

- Sie ist die konsequente Umsetzung des von Etz und Griepp eingeführten Konzepts des Staged Repair.

- Sie wird als Präventivtherapie zur Prophylaxe des ischämischen Rückenmarkschadens sowohl vor offen-chirurgischer als auch vor endovaskulärer Therapie angewendet.

- Durch MISACE soll das Risiko der Rückenmarkischämie nach komplexen Rekonstruktionen der thorakoabdominellen Aorta drastisch reduziert, möglicherweise klinisch sogar eliminiert werden.
Aorten-Stent-Eingriff zur Endoleak-Prophylaxe [39]. Besonders die Rate an Endoleak-Typ 2 nach StentgraftImplantation ist sehr hoch und die Post-Stent-Endoleak-Therapie ist technisch aufwendig und schwierig. Darum stellt die superselektive Coil- und Plug-Okklusion der vom Stent überdeckten Segmentarterien bzw. der A. mesenterica inferior eine mögliche präventive Therapieoption dar [40].

\section{Studienlage}

Erste experimentelle und klinische Studien konnten zeigen, dass die Präkonditionierung mittels MISACE einfach durchführbar ist $[38,41]$. MISACE könnte als präventive, minimalinvasive Methode zur Rückenmarkprotektion die Inzidenz des postoperativen Rückenmarkschadens drastisch senken. Deshalb konzipierten Etz u. Mitarb. eine prospektive, randomisierte und multizentrische Studie. „PAPA_Artis“ (Paraplegia Prevention in aortic Aneurysm Repair by thoracoabdominal Staging with minimally-invasive segmental Artery Coil-Embolization [MISACE]) startete im Januar 2017. Sie wird ab 2018 mit der Rekrutierung von Patienten mit thorakoabdominellem Aortenaneurysma an 16 international renommierten und auf Aortenpathologien spezialisierten Zentren beginnen. Eine Erweiterung von PAPA_Artis im Rahmen einer Förderung durch die DFG (Deutsche Forschungsgemeinschaft) an weiteren 12 deutschen Zentren ist beantragt und soll durch zusätzlich Patienten mit Aneurysmen des Typs Crawford III einschließen. Das Studienvorhaben ist auf eine Dauer von 5 Jahren ausgelegt. Die Studie beinhaltet sowohl die offen-chirurgische als auch die endovaskuläre Therapie des thorakoabdominellen Aortenaneurysmas. Sie wird möglicherweise sogar erstmals einen Vergleich zwischen der offenen und der endovaskulären Behandlung ermöglichen.

\section{Limitationen und Risiken}

Zur weiteren Optimierung der Methode ist es notwendig, potenzielle verfahrensassoziierte Risiken und Limitationen genauer zu untersuchen.

Die potenziell schwerwiegendste Komplikation stellt die iatrogene Rückenmarkschädigung dar. Diese kann durch den Verschluss einer kritischen Anzahl von Segmentarterien oder aufgrund direkter Gefäßschädigung ausgelöst werden, z. B. durch Dissektion oder Ruptur einer für die Rückenmarkperfusion relevanten Segmentarterie. Auch die Translokation thrombotischen Materials im Rahmen der Kathetermanipulation in der aneurysmatisch erweiterten Aorta - oder durch unzureichendes Coiling mit residualem distalen Fluss kann eine distale Embolisation mit irreversibler ischämischer Organschädigung zur Folge haben. Diese ist in umfangreichen Pilotserien bisher jedoch nicht eingetreten. 
Eine mögliche Methode zur reversiblen Testung des akuten Effekts der Segmentarterienokklusion stellt die Ballonokklusion der zu verschließenden Zielgefäße dar. Mit dem Ballonkatheter in Position können dann etwaige neurologische Symptome evaluiert werden. Diese Methode könnte hilfreiche Informationen über die maximale Anzahl zu verschließender Segmentarterien geben, ohne eine irreversible Rückenmarkschädigung auszulösen [19]. Das Risiko, durch dieses Manöver selbst eine Verletzung der Segmentarterien zu verursachen, ist neben der Schwierigkeit der Intubation von Segmentarterien mittels Ballonkatheter ein Grund dafür, warum das 2-zeitige Vorgehen sinnvoller erscheint.

\section{Merke}

Ein zentrales und aufgrund der gegenwärtigen Datenlage noch nicht lösbares Problem der Präkonditionierung besteht im optimalen Timing zwischen den einzelnen MISACE-Sitzungen und der definitiven Therapie.

Die Vorteile der Rückenmarkprotektion durch MISACE müssen gegen das Risiko einer akut-letalen Aneurysmaruptur (oder -dissektion) im Intervall abgewogen werden. Eine mögliche Handlungsweise ist, MISACE während der Beobachtungsphase von Patienten mit präkritischem Aortendiameter durchzuführen. So lässt sich das Risiko einer spontanen Komplikation im Intervall minimieren.

\section{Interessenkonflikt}

Die Autoren geben an, dass keine Interessenkonflikte bestehen.

\section{Über die Autoren}

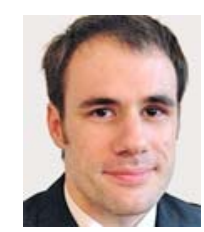

\section{Konstantin von Aspern}

Dr. med.; 2004 - 2010 Studium der Humanmedizin an der Universität Leipzig und der Universität Bergen, Norwegen. Seit 2011 in Facharztausbildung für Herzchirurgie am Herzzentrum Universität Leipzig. Forschungsgruppenleiter der Heisenberg Projektgruppe „Rückenmarkprotektion“ am Sächsischen Inkubator für Klinische Translation (SIKT) der Universität Leipzig. Schwerpunkte: Rückenmarkprotektion und nichtinvasives Monitoring in der Aortenchirurgie

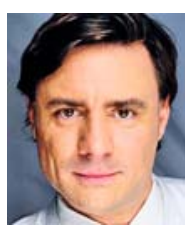

\section{Christian D. Etz}

Prof. Dr. med.; 1994-2001 Studium der Humanmedizin an der Universität Leipzig und der Universität Münster. Ab 2001 Facharztausbildung für Herzchirurgie am Universitätskrankenhaus Münster. Zwischen 2005-2008 Leiter des Forschungslabors im Mount Sinai Medical Center, New York. Ab 2008 Assistant Research Professor, Mount Sinai Medical Center, New York. Seit 2010 am Herzzentrum Universität Leipzig. Habilita- tion 2012 und seither klinischer Oberarzt - Bereich Aortenchirurgie. 2016 Heisenberg Professor für Aortenchirurgie (DFG). Direktor des Sächsischen Inkubators für Klinische Translation (SIKT) der Universität Leipzig. Schwerpunkte: neue Strategien in der Aortenchirurgie, präventive Rückenmarkprotektion; Themenkomplex bikuspide Aortenklappe; translationale Herz- und Kreislaufforschung

Korrespondenzadresse

Prof. Dr. med. Christian D. Etz

Herzzentrum Universität Leipzig

Abteilung für Herz- und Thoraxchirurgie

Struempellstr. 39

04289 Leipzig

E-Mail: Christian.Etz@medizin.uni-leipzig.de

Literatur

[1] Kalder ], Kotelis D, Jacobs M]. Thorakoabdominelles Aortenaneurysma. Gefäßchirurgie 2016; 21: 115-128

[2] Hiratzka LF, Bakris GL, Beckman JA et al. 2010 ACCF/AHA/ AATS/ACR/ASA/SCA/SCAI/SIR/STS/SVM guidelines for the diagnosis and management of patients with thoracic aortic disease. Circulation 2010; 121 : e266-e369

[3] Koeppel TA, Grainer A, Jacobs MJ. DGG Leitlinie - Thorakale und Thorakoabdominelle Aortenaneurysmen. AachenMaastricht: Europäisches Gefäßzentrum; 2010

[4] Etz CD, Halstead JC, Spielvogel D et al. Thoracic and thoracoabdominal aneurysm repair: Is reimplantation of spinal cord arteries a waste of time? Ann Thorac Surg 2006; 82: $1670-1677$

[5] Adamkiewicz A. Die Blutgefäße des menschlichen Rückenmarkes. I. Teil: Die Gefäße der Rückenmarkssubstanz. Sitzung 3. November 1881. Krakau: Institut für experimentelle Pathologie der KK Universität Krakau; 1881

[6] Wynn M, Acher C, Marks E et al. The effect of intercostal artery reimplantation on spinal cord injury in thoracoabdominal aortic aneurysm surgery. J Vasc Surg 2016; 64 : 289 296

[7] Lazorthes G, Gouaze A, Zadeh JO et al. Arterial vascularization of the spinal cord. Recent studies of the anastomotic substitution pathways. J Neurosurg 1971; $35: 253-262$

[8] Etz CD, Kari FA, Mueller CS et al. The collateral network concept: remodeling of the arterial collateral network after experimental segmental artery sacrifice. J Thorac Cardiovasc Surg 2011; $141: 1029-1036$

[9] Etz CD, Kari FA, Mueller CS et al. The collateral network concept: a reassessment of the anatomy of spinal cord perfusion. J Thorac Cardiovasc Surg 2011; 141 : 1020-1028

[10] Kari FA, Beyersdorf F. Aortic surgery and spinal collateral flow: a call for structured approaches to functional characterization of the intraspinal collateral system. J Thorac Cardiovasc Surg 2015; 149: $1675-1680$

[11] Etz CD, Homann TM, Plestis KA et al. Spinal cord perfusion after extensive segmental artery sacrifice: Can paraplegia be prevented? Eur J Cardiothorac Surg 2007; 31: 643-648

[12] Etz CD, Luehr M, Kari FA et al. Paraplegia after extensive thoracic and thoracoabdominal aortic aneurysm repair: 
Does critical spinal cord ischemia occur postoperatively? ] Thorac Cardiovasc Surg 2008; 135: 324 - 330

[13] Etz CD, Zoli S, Mueller CS et al. Staged repair significantly reduces paraplegia rate after extensive thoracoabdominal aortic aneurysm repair. J Thorac Cardiovasc Surg 2010; 139: $1464-1472$

[14] Maurel B, Delclaux N, Sobocinski ] et al. The impact of early pelvic and lower limb reperfusion and attentive peri-operative management on the incidence of spinal cord ischemia during thoracoabdominal aortic aneurysm endovascular repair. Eur J Vasc Endovasc Surg 2015; 49: 248 - 254

[15] Pillai JB, Pellet $Y$, Panagopoulos $G$ et al. Somatosensoryevoked potential-guided intercostal artery reimplantation in thoracoabdominal aortic aneurysm surgery. Innovations (Phila) 2013; 8: 302-306

[16] Etz CD, von Aspern K, Gudehus S et al. Near-infrared spectroscopy monitoring of the collateral network prior to, during, and after thoracoabdominal aortic repair: a pilot study. Eur J Vasc Endovasc Surg 2013; 46: 651-656

[17] Boezeman RP, van Dongen EP, Morshuis WJ et al. Spinal near-infrared spectroscopy measurements during and after thoracoabdominal aortic aneurysm repair: a pilot study. Ann Thorac Surg 2015; 99: 1267-1274

[18] von Aspern K, Haunschild J, Hoyer A et al. Non-invasive spinal cord oxygenation monitoring: validating collateral network near-infrared spectroscopy for thoracoabdominal aortic aneurysm repair. Eur J Cardiothorac Surg 2016; 50: $675-683$

[19] von Aspern K, Luehr M, Mohr FW et al. Spinal cord protection in open- and endovascular thoracoabdominal aortic aneurysm repair: critical review of current concepts and future perspectives. J Cardiovasc Surg (Torino) 2015; 56: $745-749$

[20] Luehr M, Bachet J, Mohr FW et al. Modern temperature management in aortic arch surgery: the dilemma of moderate hypothermia. Eur J Cardiothorac Surg 2014; 45: 27-39

[21] Acher C, Acher CW, Marks E et al. Intraoperative neuroprotective interventions prevent spinal cord ischemia and injury in thoracic endovascular aortic repair. J Vasc Surg 2016; 63: $1458-1465$

[22] Fedorow CA, Moon MC, Mutch WA et al. Lumbar cerebrospinal fluid drainage for thoracoabdominal aortic surgery: rationale and practical considerations for management. Anesth Analg 2010; 111: 46-58

[23] Coselli JS, LeMaire SA, Koksoy C et al. Cerebrospinal fluid drainage reduces paraplegia after thoracoabdominal aortic aneurysm repair: results of a randomized clinical trial. J Vasc Surg 2002; 35: 631-639

[24] Dardik A, Perler BA, Roseborough GS et al. Subdural hematoma after thoracoabdominal aortic aneurysm repair: an underreported complication of spinal fluid drainage? J Vasc Surg 2002; 36: $47-50$

[25] Wynn MM, Sebranek J, Marks E et al. Complications of spinal fluid drainage in thoracic and thoracoabdominal aortic aneurysm surgery in 724 patients treated from 1987 to 2013. J Cardiothorac Vasc Anesth 2015; 29: 342 - 350

[26] Estrera AL, Sheinbaum R, Miller CC et al. Cerebrospinal fluid drainage during thoracic aortic repair: safety and current management. Ann Thorac Surg 2009; 88: 9-15; discussion 15

[27] Tanaka $\mathrm{H}$, Minatoya $\mathrm{K}$, Matsuda $\mathrm{H}$ et al. Embolism is emerging as a major cause of spinal cord injury after descending and thoracoabdominal aortic repair with a contemporary approach: magnetic resonance findings of spinal cord injury. Interact Cardiovasc Thorac Surg 2014; 19: 205 - 210

[28] Nardone R, Pikija S, Mutzenbach JS et al. Current and emerging treatment options for spinal cord ischemia. Drug Discov Today 2016; 21: $1632-1641$

[29] Wynn MM, Acher CW. A modern theory of spinal cord ischemia/injury in thoracoabdominal aortic surgery and its implications for prevention of paralysis. J Cardiothorac Vasc Anesth 2014; 28: 1088-1099

[30] Tschop J, Czerner S, Nuscheler M et al. Epidural cooling. Neuroprotective treatment of thoracoabdominal aortic aneurysms. Anaesthesist 2008; 57: 988-997

[31] Shimizu H, Mori A, Yamada T et al. Regional spinal cord cooling using a countercurrent closed-lumen epidural catheter. Ann Thorac Surg 2010; 89: 1312-1313

[32] Acher C. It is not just assisted circulation, hypothermic arrest, or clamp and sew. J Thorac Cardiovasc Surg 2010; 140: (Suppl. 06): S136-S141; discussion S142-S146

[33] Estrera AL, Sheinbaum R, Miller CC3rd et al. Neuromonitorguided repair of thoracoabdominal aortic aneurysms. J Thorac Cardiovasc Surg 2010; 140: (Suppl. 06): S131 - S135; discussion S142-S146

[34] Weiss AJ, Pawale A, Griepp RB et al. Deep versus mild hypothermia during thoracoabdominal aortic surgery. Ann Cardiothorac Surg 2012; 1: 329-333

[35] Coselli JS, de la Cruz KI, Preventza O et al. Extent II thoracoabdominal aortic aneurysm repair: how I do it. Semin Thorac Cardiovasc Surg 2016; 28: 221-237

[36] David N, Roux N, Douvrin F et al. Aortic aneurysm surgery: long-term patency of the reimplanted intercostal arteries. Ann Vasc Surg 2012; 26: 839-844

[37] Kasprzak PM, Gallis K, Cucuruz B et al. Editor's choice temporary aneurysm sac perfusion as an adjunct for prevention of spinal cord ischemia after branched endovascular repair of thoracoabdominal aneurysms. Eur J Vasc Endovasc Surg 2014; 48: 258-265

[38] Etz CD, Debus ES, Mohr FW et al. First-in-man endovascular preconditioning of the paraspinal collateral network by segmental artery coil embolization to prevent ischemic spinal cord injury. J Thorac Cardiovasc Surg 2015; 149: 1074 1079

[39] Parry DJ, Kessel DO, Robertson I et al. Type II endoleaks: predictable, preventable, and sometimes treatable? J Vasc Surg 2002; 36: $105-110$

[40] Kasirajan K, Matteson B, Marek JM et al. Technique and results of transfemoral superselective coil embolization of type II lumbar endoleak. J Vasc Surg 2003; 38: 61-66

[41] Geisbusch S, Stefanovic A, Koruth JS et al. Endovascular coil embolization of segmental arteries prevents paraplegia after subsequent thoracoabdominal aneurysm repair: an experimental model. J Thorac Cardiovasc Surg 2014; 147: $220-226$

Bibliografie

DOI http://dx.doi.org/10.1055/s-0043-102158

Gefäßmedizin Scan 2017; 04: 41-56

(c) Georg Thieme Verlag KG Stuttgart · New York

ISSN 2197-5922 


\section{Punkte sammeln auf CME.thieme.de}

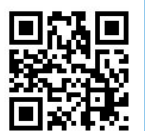

Diese Fortbildungseinheit ist 12 Monate online für die Teilnahme verfügbar. Sollten Sie Fragen zur Online-Teilnahme haben, finden Sie unter http://cme.thieme.de/hilfe eine ausführliche Anleitung. Wir wünschen viel Erfolg beim Beantworten der Fragen!

Unter eref.thieme.de/ZZX8LKI oder über den QR-Code kommen Sie direkt zum Artikel zur Eingabe der Antworten.

VNR 2760512017152370666

\section{Frage 1}

Bei welchen Patienten steigt das Komplikationsrisiko bei thorakoabdominellen Aortenaneurysmen exponentiell?

A bei Patienten über 70 Jahren

B bei Patienten mit einem maximalen Aortendurchmesser von über $6 \mathrm{~cm}$

C bei Patienten mit einem mittleren arteriellen Druck über $100 \mathrm{mmHg}$

D bei Patienten mit Diabetes mellitus als Nebendiagnose

E bei Patienten mit vielen patenten Segmentarterien

\section{Frage 2}

Welche der folgenden Aussagen gilt für das therapieassoziierte Risiko der Rückenmarkischämie bei thorakoabdominellen Aortenaneurysmen (Crawford-Typ II)?

A Es beträgt bis zu 7\% nach offen-chirurgischem Aortenersatz.

B Es beträgt unter 3 \% für die endovaskuläre StentgraftImplantation.

C Es ist für die chirurgische Therapie deutlich höher als für die endovaskuläre.

D Es hängt nicht vom postoperativen Management ab und kann durch Senkung des arteriellen Blutdrucks verringert werden.

E Es beträgt auch heutzutage noch bis zu $18 \%$ und ist für chirurgische und endovaskuläre Therapien vergleichbar.

\section{Frage 3}

Welche der folgenden Maßnahmen ist nicht im peri- und postoperativen Management zur Rückenmarkprotektion enthalten?

A hochnormales Blutdruckmanagement

B zerebrospinale Liquordrainage

C Hyperthermie

D adäquate Volumensubstitution

E Vermeidung der intravenösen Gabe von Nitraten und $\alpha$-Blockern

\section{Frage 4}

Von welcher Voraussetzung geht das neue Konzept der Rückenmarkperfusion aus?

A Eine einzige Arterie (A. Adamkiewicz) stellt die komplette Rückenmarkperfusion sicher.

B Es existiert ein umfangreiches paraspinales Kollateralnetzwerk, das die Rückenmarkperfusion auch segmentübergreifend sicherstellen kann.

C Das paraspinale Kollateralnetzwerk kann sich nicht auf Änderungen im Blutfluss einstellen.

D Der Zufluss zum Kollateralnetzwerk erfolgt allein aus den aortalen Segmentarterien.

E Der Verschluss aller Segmentarterien in einem Schritt ist mit dem geringsten Risiko einer spinalen Ischämie assoziiert.

\section{Frage 5}

Welches Konzept konnte die Rate an Paraplegien nach ausgedehnten Operationen an der thorakoabdominellen Aorta drastisch senken?

A Reimplantation der Segmentarterien

B Operation bei normaler Körpertemperatur

C mehrzeitiges Vorgehen (Staged Repair)

D lokale, kontinuierliche Applikation normothermer isotoner Kochsalzlösung

E Gabe von Kortikosteroiden zur Inflammationshemmung

\section{Frage 6}

Welche der folgenden Methoden gehört nicht zu denjenigen, die zur intraoperativen neurologischen Überwachung angewendet werden?

A Messung von MEP (motorisch evozierten Potenzialen)

B cnNIRS (Nahinfrarotspektroskopie des Kollateralnetzwerks)

C invasive Laser-Doppler-Flussmessung des Rückenmarks

D regelmäßige klinische Untersuchung und Befragung des Patienten (auch bei Interventionen in Lokalanästhesie)

E Messung von SSEP (somatosensibel evozierten Potenzialen) 


\section{Punkte sammeln auf CME.thieme.de}

Fortsetzung ...

\section{Frage 7}

Welche der folgenden Vorgaben zählt nicht zu den Empfehlungen der Rückenmarkprotektion nach COPS-Schema bei neurologischem Defizit?

A Hämoglobingehalt über $10 \mathrm{~g} / \mathrm{dl}$

B Blutdruck systolisch über $130 \mathrm{mmHg}$ und hochnormaler Mitteldruck

C Gabe von Nitraten zur Verbesserung der Durchblutung

D Liquordrainage auf einen intrakraniellen Druck unter $7 \mathrm{mmHg}$

E arterielle Sauerstoffsättigung über $97 \%$

\section{Frage 8}

Welche der folgenden Maßnahmen gehört zu den intraoperativen Techniken der Rückenmarkprotektion bei TEVAR (Thoracic endovascular aortic Repair)?

A frühzeitige Freigabe der Beckenachse durch Zurückziehen der Schleusen

B lokale, intraaortale Infusion vasodilatatorischer Substanzen

C selektiver Verschluss von Segmentarterien und/oder der A. mesenterica inferior

D aktives Wärmen des Patienten auf mindestens $37^{\circ} \mathrm{C}$ Körpertemperatur

E Einsatz großkalibriger Schleusen

\section{Frage 9}

Welche der folgenden Aussagen trifft zu?

A Die TASP (temporäre Aneurysmaperfusion) ist eine Methode zur Rückenmarkprotektion beim offenchirurgischen Aortenersatz.

B Bei Stentgrafts in der Aorta mit einer Länge von $10 \mathrm{~cm}$ wird die Anlage eines lumbalen Liquordrainagekatheters zur Rückenmarkprotektion empfohlen.

C Blutungskomplikationen mit neurologischer Symptomatik durch Anlage eines lumbalen Liquordrainagekatheters sind häufig (in mehr als $20 \%$ der Fälle).

D Ein positiver Effekt der medikamentösen Therapien zur Rückenmarkprotektion wurde in klinischen Studien nachgewiesen.

E Vor Anlage eines lumbalen Katheters zur Liquordrainage sollten die Gerinnungsparameter ausgeglichen und Heparin pausiert sein.

\section{Frage 10}

Welche der folgenden Aussagen über die MISACE (minimalinvasive Segmentarterien-Coil-/Plug-Embolisation) ist richtig?

A Grundlage der Methode ist eine Präkonditionierung des intraspinalen Gefäßsystems des Rückenmarks.

B Die Methode basiert auf dem Konzept des Staged Repair zur Rückenmarkprotektion.

C MISACE wird aktuell vor allem bei symptomatischen Patienten mit dringlicher Operationsindikation angewendet.

D MISACE erhöht das Risiko von Endoleaks (Typ 2) nach TEVAR.

E Eine Translokation unterdimensionierter Coils in den distalen Abschnitt von Segmentarterien und A. mesenterica inferior ist unkritisch. 